\title{
Difference Equation for A Population Model
}

\author{
MAMI SUZUKI \\ Department of Mathematics, Faculty of Informatics, Teikyo Heisei University, \\ Otani 2289-23, Uruido Ichihara-shi, Chiba, 290-0193, Japan
}

(Received 11 August 1999)

In this paper we consider a difference equation of the form

$$
u(t+2)=\alpha u(t+1)+\beta \frac{u(t+1)-\alpha u(t)}{\alpha u(t)}, \text { for } t>-\infty,
$$

\begin{abstract}
seems to be a general statement of a relative socio-spatial dynamics. Indeed this equation is one of "population model". We investigate behavior of solutions and expression of analytic general solutions of this model.
\end{abstract}

Keywords: Difference equation, Dynamics model, Analytic solution

\section{INTRODUCTION}

Recently many kinds of population models are formulated with difference equations $[1,3,4]$. Here we consider the following difference equation:

$$
u(t+2)=\alpha u(t+1)+\beta \frac{u(t+1)-\alpha u(t)}{\alpha u(t)},
$$

where $\alpha=1+r$, in which $r$ is the net (births minus death) endogenous population (stock) growth rate, and the second term is a function depicting net immigration at $t+1$, in turn a function of a "momentum" to grow from $t$ to $t+1$. So that we assume that $\alpha>0(r>-1)$ and $\beta>0$ in (1.1).

The model (1.1) was proposed by Prof. Dimitrios Dendrinos [2]. We are interested in it because it is a simple three-time-period model. Let

$$
u(t+2)=u_{1}(t+2)+u_{2}(t+2)
$$

where

$$
\begin{aligned}
& u_{1}(t+2)=\alpha u(t+1), \\
& u_{2}(t+2)=\beta \frac{u(t+1)-\alpha u(t)}{\alpha u(t)} .
\end{aligned}
$$

In this model $\alpha$ and $\beta$ are presumably independent of the time $t$. So we assume that $\alpha$ and $\beta$ are constants. $u_{1}(t+2)$ is a term for endogenous population growth rate from $t+1$ to $t+2$, and $u_{2}(t+2)$ due to net in-migration rate. Indeed

$$
\begin{aligned}
u_{1}(t+2) & =\alpha u(t+1)=\alpha\left\{u_{1}(t+1)+u_{2}(t+1)\right\}, \\
u_{2}(t+2) & =\beta \frac{u(t+1)-\alpha u(t)}{\alpha u(t)} \\
& =\beta \frac{u(t+1)-u_{1}(t+1)}{u_{1}(t+1)} \\
& =\beta \frac{u_{2}(t+1)}{u_{1}(t+1)},
\end{aligned}
$$


where $u_{1}(t+1)$ is the endogenous population growth rate from $t$ to $t+1$, and $u_{2}(t+1)$ due to net in-migration rate.

In this paper we will study it, at first, from the viewpoint of (real) state-space analysis, especially of stability properties as $t \rightarrow \infty$, for positive solutions. Next we study it from the complex analytic view point.

We may write (1.1) as:

$$
\begin{aligned}
& u(t+2)-\alpha u(t+1) \\
& \quad=\frac{c}{u(t)}\{u(t+1)-\alpha u(t)\}, \quad c=\frac{\beta}{\alpha} .
\end{aligned}
$$

When $\alpha \neq 1,(1.1)$ admits the unique equilibrium value $c=\beta / \alpha$. When $\alpha=1$, any value may be the equilibrium.

\section{STABILITY PROPERTIES OF POSITIVE SOLUTIONS}

In this section, we suppose that $u(t)$ is a solution of (1.1) which is positive for $t \geqq t_{0}$, with the initial values $u\left(t_{0}\right)$ and $u\left(t_{0}+1\right)$.

We say $u(t)$ is stable if there are positive constants $L_{1}, L_{2}$ such that

$$
0<L_{1} \leqq u\left(t_{0}+n\right) \leqq L_{2} \quad \text { for } n \in \mathbb{N}
$$

$u(t)$ is asymptotically stable if there is $b_{0}$ such that, for any $\epsilon>0$ there is $n_{0} \in \mathbb{N}$ such that

$$
\begin{array}{r}
\left|u\left(t_{0}+n\right)-b_{0}\right|<\epsilon \quad \text { for } n \geqq n_{0}, \\
\text { i.e. } \quad u\left(t_{0}+n\right) \rightarrow b_{0}(n \rightarrow \infty) .
\end{array}
$$

We write (1.1) as

$$
\begin{aligned}
& u(t+2)-\alpha u(t+1) \\
& \quad=\frac{c}{u(t)}(u(t+1)-\alpha u(t)), \quad c=\frac{\beta}{\alpha} .
\end{aligned}
$$

It is easy to see that, if $\lim _{n \rightarrow \infty} u\left(t_{0}+n\right)=u_{0}$ for some $t_{0}$ and $\alpha \neq 1$, then we must have $u_{0}=c$.

\subsection{When $\alpha>1$}

Suppose $u\left(t_{0}+1\right)-\alpha u\left(t_{0}\right) \geqq 0$ for some $t_{0}$. Then we have $u\left(t_{0}+2\right)-\alpha u\left(t_{0}+1\right) \geqq 0$ by (2.1). Hence

$$
\begin{aligned}
u(t+n) & \geqq \alpha u(t+n-1) \geqq \cdots \\
& \geqq \alpha^{n-1} u(t+1) \geqq \alpha^{n} u(t)>0 .
\end{aligned}
$$

Thus we have $u\left(t_{0}+n\right) \rightarrow \infty(n \rightarrow \infty)$ and the solution $u(t)$ is not stable. So, we assume that $u(t+1)-$ $\alpha u(t)<0$ for any $t \geqq 0$.

(i) Suppose $u\left(t_{0}\right) \geqq c$. If $u\left(t_{0}+1\right)>u\left(t_{0}\right)$, then

$$
\begin{aligned}
0 & \leqq \alpha u\left(t_{0}+1\right)-u\left(t_{0}+2\right) \\
& =\frac{c}{u\left(t_{0}\right)}\left\{\alpha u\left(t_{0}\right)-u\left(t_{0}+1\right)\right\} \\
& \leqq \alpha u\left(t_{0}\right)-u\left(t_{0}+1\right)
\end{aligned}
$$

So we have

$0<\alpha\left\{u\left(t_{0}+1\right)-u\left(t_{0}\right)\right\} \leqq u\left(t_{0}+2\right)-u\left(t_{0}+1\right)$.

Since $u\left(t_{0}+1\right)>u\left(t_{0}\right) \geqq c$, we have

$$
\begin{aligned}
& u\left(t_{0}+n+1\right)-u\left(t_{0}+n\right) \\
& \quad \geqq \alpha^{n}\left\{u\left(t_{0}+1\right)-u\left(t_{0}\right)\right\}>0 .
\end{aligned}
$$

Therefore we have $u\left(t_{0}+n\right) \rightarrow \infty$ and $u(t)$ is not stable. So we must have that $u(t+1) \leqq u(t)$ whenever $u(t) \geqq c$. If otherwise, i.e. if there is $t_{0}$ such that $u\left(t_{0}\right) \geqq c$ and $u\left(t_{0}+1\right)>u\left(t_{0}\right)$, then $u(t)$ is not stable. If $u(t)$ is stable and $u(t) \geqq c$ for any $t$, then $c$ is an asymptotically stable equilib. point $: u(t) \downarrow c$.

(ii) Suppose $u\left(t_{0}\right)<c$ for some $t_{0}$. If $u\left(t_{0}+1\right)<$ $u\left(t_{0}\right)$, then

$$
\begin{aligned}
& u\left(t_{0}+2\right)-u\left(t_{0}+1\right)<\alpha\left(u\left(t_{0}+1\right)-u\left(t_{0}\right)\right)<0, \\
& u\left(t_{0}+2\right)<u\left(t_{0}+1\right)<u\left(t_{0}\right) \leqq c,
\end{aligned}
$$

and

$$
\begin{aligned}
& u\left(t_{0}+n\right) \\
& \quad<\left(1+\alpha+\cdots+\alpha^{n-1}\right)\left\{u\left(t_{0}+1\right)-u\left(t_{0}\right)\right\}<0 .
\end{aligned}
$$


Thus $u\left(t_{0}+n\right)$ tend to $-\infty$, and $u(t)$ is not stable. Therefore, if $u(t)$ is stable, we must have

$$
\begin{array}{ll}
u(t+1) \leqq u(t) & \text { whenever } u(t)>c, \\
u(t+1) \geqq u(t) \quad \text { whenever } u(t)<c .
\end{array}
$$

Conversely, if (2.3) holds, then $u(t) \rightarrow c$ as $t \rightarrow \infty$. Therefore we see that $u(t)$ is stable if and only if $u(t)$ is asymptotically stable, and $c$ is the only stable equilib. point.

In Section 4, we have really exist stable positive solutions when $\alpha>1$.

\subsection{When $\alpha<1$}

Suppose $u\left(t_{0}+1\right)-\alpha u\left(t_{0}\right) \leqq 0$, for some $t_{0}$. Then by (2.1) we have

$$
\begin{aligned}
0<u\left(t_{0}+n\right) & \leqq \alpha u\left(t_{0}+n-1\right) \leqq \cdots \\
& \leqq \alpha^{n-1} u\left(t_{0}+1\right) \leqq \alpha^{n} u\left(t_{0}\right) .
\end{aligned}
$$

Since $\alpha<1$, we get $u\left(t_{0}+n\right) \rightarrow 0$ and the population tends to death. So we may assume that

$$
u(t+1)-\alpha u(t)>0 \text { for any } t .
$$

(i) When $u\left(t_{0}\right) \leq c$ for some $t_{0}$. We have

$$
\begin{aligned}
\alpha u\left(t_{0}+1\right)-u\left(t_{0}+2\right) & =\frac{c}{u\left(t_{0}\right)}\left\{\alpha u\left(t_{0}\right)-u\left(t_{0}+1\right)\right\} \\
& \leqq \alpha u\left(t_{0}\right)-u\left(t_{0}+1\right)<0,
\end{aligned}
$$

hence

$$
\alpha\left(u\left(t_{0}+1\right)-u\left(t_{0}\right)\right) \leqq u\left(t_{0}+2\right)-u\left(t_{0}+1\right) .
$$

(i-a) Suppose $u\left(t_{0}+1\right)-u\left(t_{0}\right) \geqq 0 . \quad$ Then $u\left(t_{0}+2\right)-u\left(t_{0}+1\right) \geqq 0$. Suppose there is $k_{0}$ such that $u\left(t_{0}+k\right) \leqq c \quad\left(0 \leqq k<k_{0}\right)$ and $u\left(t_{0}+k_{0}\right)>c$. (If there is no such $k_{0}$, we have $u\left(t_{0}+k\right) \uparrow c$.) Then

$$
\begin{aligned}
u\left(t_{0}+\right. & \left.k_{0}+1\right) \\
= & \alpha u\left(t_{0}+k_{0}\right)+\frac{c}{u\left(t_{0}+k_{0}-1\right)} u\left(t_{0}+k_{0}\right)-\alpha c \\
> & \alpha u\left(t_{0}+k_{0}\right)+\frac{c}{u\left(t_{0}+k_{0}-1\right)} u\left(t_{0}+k_{0}\right) \\
& -\alpha u\left(t_{0}+k_{0}\right) \\
= & \frac{c}{u\left(t_{0}+k_{0}-1\right)} u\left(t_{0}+k_{0}\right) \geqq u\left(t_{0}+k_{0}\right) .
\end{aligned}
$$

From (2.1) we have, using

$$
\begin{gathered}
\frac{c}{u\left(t_{0}+k_{0}\right)}-1 \leqq 0, \\
\frac{1}{\alpha}\left\{u\left(t_{0}+k_{0}+2\right)-u\left(t_{0}+k_{0}+1\right)\right\} \\
=\left\{1+\frac{1}{\alpha}\left(\frac{c}{u\left(t_{0}+k_{0}\right)}-1\right)\right\} u\left(t_{0}+k_{0}+1\right)-c \\
=\frac{u\left(t_{0}+k_{0}+1\right)}{\alpha}\left(\frac{c}{u\left(t_{0}+k_{0}\right)}-1\right) \\
\quad+u\left(t_{0}+k_{0}+1\right)-c \\
\leqq u\left(t_{0}+k_{0}\right)\left(\frac{c}{u\left(t_{0}+k_{0}\right)}-1\right) \\
+u\left(t_{0}+k_{0}+1\right)-c \\
=u\left(t_{0}+k_{0}+1\right)-u\left(t_{0}+k_{0}\right) .
\end{gathered}
$$

So we have

$$
\begin{aligned}
& u\left(t_{0}+k_{0}+2\right)-u\left(t_{0}+k_{0}+1\right) \\
& \quad \leqq \alpha\left\{u\left(t_{0}+k_{0}+1\right)-u\left(t_{0}+k_{0}\right)\right\} .
\end{aligned}
$$

If $u\left(t_{0}+k_{0}+2\right)-u\left(t_{0}+k_{0}+1\right) \geqq 0, \quad$ we may repeat the same argument. If we assume $u\left(t_{0}+k_{0}+n+1\right)-u\left(t_{0}+k_{0}+n\right) \geqq 0$ for any $n \geqq 1$, we would have

$$
\begin{aligned}
u\left(t_{0}+\right. & \left.k_{0}+n\right) \\
< & \left(\alpha^{n-1}+\alpha^{n-2}+\cdots+\alpha+1\right)\left\{u\left(t_{0}+k_{0}+1\right)\right. \\
& \left.\quad-u\left(t_{0}+k_{0}\right)\right\}+u\left(t_{0}+k_{0}\right) \\
= & \frac{1-\alpha^{n}}{1-\alpha}\left(u\left(t_{0}+k_{0}+1\right)-u\left(t_{0}+k_{0}\right)\right) \\
\quad & +u\left(t_{0}+k_{0}\right),
\end{aligned}
$$

and

$$
\begin{aligned}
& u\left(t_{0}+k_{0}+n\right) \uparrow u_{0} \\
& \quad \leqq \frac{1}{1-\alpha}\left\{u\left(t_{0}+k_{0}+1\right)-\alpha u\left(t_{0}+k_{0}\right)\right\}<\infty .
\end{aligned}
$$

Obviously $u_{0} \geqq u\left(t_{0}+k_{0}\right)>c$, which is a contradiction since $u\left(t_{0}+n\right) \rightarrow u_{0}$ and we must have $u_{0}=c$. Hence there is $n_{0}$ such that $u\left(t_{0}+k_{0}+n\right) \leqq$ $u\left(t_{0}+k_{0}+n+1\right) \quad\left(n<n_{0}\right) \quad$ and $u\left(t_{0}+k_{0}+n_{0}\right)>$ $u\left(t_{0}+k_{0}+n_{0}+1\right)$. 
(i-b) Suppose $u\left(t_{0}+1\right)<u\left(t_{0}\right) \leqq c$. If $u\left(t_{0}+n\right) \leqq$ $u\left(t_{0}+n-1\right)(n \geqq 1)$, then $u\left(t_{0}+n\right) \downarrow u_{0}$ and $u_{0}$ cannot be positive. Hence $u\left(t_{0}+n\right) \downarrow 0$.

If there is $k_{0}$ such that $u\left(t_{0}+k\right) \leqq u\left(t_{0}+k-1\right)$ $\left(k<k_{0}\right)$ and $u\left(t_{0}+k_{0}\right)>u\left(t_{0}+k_{0}-1\right)$, we have from (2.1), using

$$
\begin{gathered}
\frac{c}{u\left(t_{0}+k_{0}-1\right)}-1>0, \\
\frac{1}{\alpha}\left\{u\left(t_{0}+k_{0}+1\right)-u\left(t_{0}+k_{0}\right)\right\} \\
=\frac{u\left(t_{0}+k_{0}\right)}{\alpha}\left(\frac{c}{u\left(t_{0}+k_{0}-1\right)}-1\right) \\
+u\left(t_{0}+k_{0}\right)-c .
\end{gathered}
$$

Since $u\left(t_{0}+k_{0}\right)-\alpha u\left(t_{0}+k_{0}-1\right)>0$, we have

$$
\begin{aligned}
& \frac{1}{\alpha}\left\{u\left(t_{0}+k_{0}+1\right)-u\left(t_{0}+k_{0}\right)\right\} \\
&> u\left(t_{0}+k_{0}-1\right)\left(\frac{c}{u\left(t_{0}+k_{0}-1\right)}-1\right) \\
&+u\left(t_{0}+k_{0}\right)-c \\
&= u\left(t_{0}+k_{0}\right)-u\left(t_{0}+k_{0}-1\right) .
\end{aligned}
$$

If $u\left(t_{0}+k_{0}\right) \leqq c$, we may repeat the argument. Suppose there is $h_{0}$ such that $u\left(t_{0}+k_{0}+h\right) \leqq c\left(h<h_{0}\right)$ and $u\left(t_{0}+k_{0}+h_{0}\right)>c$. (If there is no such $h_{0}$, then $\left.u\left(t_{0}+n\right) \uparrow c.\right)$ By the above argument, we have $u\left(t_{0}+k_{0}+h_{0}+1\right)>u\left(t_{0}+k_{0}+h_{0}\right)>c$. Now we proceed to the following case (ii):

(ii) When $u\left(t_{0}\right)>c$. Then

$$
\begin{aligned}
0 & \geq \alpha u\left(t_{0}+1\right)-u\left(t_{0}+2\right) \\
& =\frac{c}{u\left(t_{0}\right)}\left\{\alpha u\left(t_{0}\right)-u\left(t_{0}+1\right)\right\} \\
& >\alpha u\left(t_{0}\right)-u\left(t_{0}+1\right),
\end{aligned}
$$

so we get

$$
\alpha\left\{u\left(t_{0}+1\right)-u\left(t_{0}\right)\right\}>u\left(t_{0}+2\right)-u\left(t_{0}+1\right) .
$$

(ii-a) Suppose $u\left(t_{0}+1\right)>u\left(t_{0}\right)>0$. If $u\left(t_{0}+n\right)>$ $u\left(t_{0}+n-1\right)(n \geq 1)$, we get

$$
\begin{aligned}
& \alpha\left\{u\left(t_{0}+n-1\right)-u\left(t_{0}+n-2\right)\right\} \\
& \quad>u\left(t_{0}+n\right)-u\left(t_{0}+n-1\right), \\
& u\left(t_{0}+n\right)-u\left(t_{0}+n-1\right)<\alpha^{n-1}\left\{u\left(t_{0}+1\right)-u\left(t_{0}\right)\right\} \\
& u\left(t_{0}+n\right)<u\left(t_{0}\right)+\frac{1-\alpha^{n}}{1-\alpha}\left\{u\left(t_{0}+1\right)-u\left(t_{0}\right)\right\} \\
& \quad<\frac{u\left(t_{0}+1\right)-\alpha u\left(t_{0}\right)}{1-\alpha},
\end{aligned}
$$

hence

$$
c<u\left(t_{0}+n\right) \uparrow u_{0}<\frac{u\left(t_{0}+1\right)}{1-\alpha},
$$

which is a contradiction. Thus there is $n_{0}$ such that

$$
\begin{gathered}
u\left(t_{0}+n\right)>u\left(t_{0}+n-1\right)\left(n<n_{0}\right) \quad \text { and } \\
u\left(t_{0}+n\right)>u\left(t_{0}+n-1\right) .
\end{gathered}
$$

Thus we proceed to the following case (ii-b).

(ii-b) Suppose $u\left(t_{0}+1\right)<u\left(t_{0}\right)$. Then $u\left(t_{0}+2\right)<$ $u\left(t_{0}+1\right)$ by (2.4). If $u\left(t_{0}+n\right) \geqq c$, then we get $u\left(t_{0}+n\right) \downarrow c$. If $u\left(t_{0}+n\right) \geqq c\left(n<n_{0}\right)$ and $u\left(t_{0}+n_{0}\right)<c$, then we come back to the case (i).

Thus we see that, when $0<\alpha<1$, we have either $u(t+n) \rightarrow 0$ or $u(t)$ is stable.

\subsection{When $\alpha=1$}

We have in this case

$$
u(t+2)-u(t+1)=\frac{c}{u(t)}(u(t+1)-u(t))
$$

Note that,

$$
\text { if } \begin{aligned}
& \lim _{n \rightarrow \infty} u\left(t_{0}+n\right)=u_{0} \neq \pm \infty, \\
& \text { then } \lim _{n \rightarrow \infty} \frac{u\left(t_{0}+n+1\right)}{u\left(t_{0}+n\right)}=1 .
\end{aligned}
$$

(i) When $u\left(t_{0}+1\right)>u\left(t_{0}\right)$. Then $u\left(t_{0}+n\right)>$ $u\left(t_{0}+n-1\right)(n \in \mathbb{N})$. If $u\left(t_{0}\right) \geqq c$, then

$$
b=\frac{c}{u\left(t_{0}+1\right)}<1 \text {. }
$$


Hence

$$
\begin{aligned}
u\left(t_{0}+n\right)-u\left(t_{0}+n-1\right) & \\
= & \frac{c}{u\left(t_{0}+n-2\right)} \cdots \frac{c}{u\left(t_{0}+1\right)} \frac{c}{u\left(t_{0}\right)} \\
& \times\left\{u\left(t_{0}+1\right)-u\left(t_{0}\right)\right\} \\
< & b^{n-2}\left\{u\left(t_{0}+1\right)-u\left(t_{0}\right)\right\}, \\
u\left(t_{0}+n\right)< & \frac{1-b^{n-1}}{1-b}\left\{u\left(t_{0}+1\right)-u\left(t_{0}\right)\right\} \\
& +u\left(t_{0}+1\right), \\
u\left(t_{0}+n\right) \uparrow u_{0} \leqq & \frac{(2-b) u\left(t_{0}+1\right)-u\left(t_{0}\right)}{1-b}, \quad\left(u_{0}>c\right) .
\end{aligned}
$$

If $u\left(t_{0}\right)<c$, then $u\left(t_{0}+2\right)-u\left(t_{0}+1\right)=c / u\left(t_{0}\right) \times$ $\left\{u\left(t_{0}+1\right)-u\left(t_{0}\right)\right\}>u\left(t_{0}+1\right)-u\left(t_{0}\right)>0 \quad(n \in \mathbb{N})$, hence there is $n_{0}$ such that $u\left(t_{0}+n_{0}\right)>c$. Hereafter $u\left(t_{0}+n\right)$ behaves as above and we get $u\left(t_{0}+n\right) \uparrow$ $u_{0}<\infty,\left(u_{0}>c\right)$.

(ii) When $u\left(t_{0}+1\right)<u\left(t_{0}\right)$. Then $u\left(t_{0}+n+1\right)-$ $u\left(t_{0}+n\right)<0$ for $u\left(t_{0}+j\right)>0$ for $0 \leqq j \leqq n-1$.

If $u\left(t_{0}\right) \leqq c$, then $u\left(t_{0}-1+n\right)<c$, hence

$$
\begin{aligned}
& u\left(t_{0}+n+2\right)-u\left(t_{0}+n+1\right) \\
& \quad=\frac{c}{u\left(t_{0}+n\right)}\left\{u\left(t_{0}+n+1\right)-u\left(t_{0}+n\right)\right\} \\
& \quad \leqq u\left(t_{0}+n+1\right)-u\left(t_{0}+n\right)<0,
\end{aligned}
$$

if $u\left(t_{0}+n\right)>0$. Thus there is $n_{0}$ such that $u\left(t_{0}+n\right)>0$ for $n<n_{0}$ and $u\left(t_{0}+n_{0}\right) \leqq 0$. Then $u\left(t_{0}+n_{0}+2\right)-$ $u\left(t_{0}+n_{0}+1\right)>0$ since $u\left(t_{0}+n_{0}+1\right)-u\left(t_{0}+n_{0}\right)<0$, and $u\left(t_{0}+n_{0}+3\right)-u\left(t_{0}+n_{0}+2\right)<0$, since $u\left(t_{0}+\right.$ $\left.n_{0}+1\right)<0$. Thus $u\left(t_{0}+n\right)$ oscillate.

If $u\left(t_{0}\right)>c$ and $u\left(t_{0}+n_{1}\right) \leqq c$ for some $n_{1}$, then from above discussion, $u\left(t_{0}+n\right)$ oscillate.

If $u\left(t_{0}\right)>c$ and $u\left(t_{0}+n\right)>c$ for all $n$, then there is a $u_{0}$ such that $u\left(t_{0}+n\right) \downarrow u_{0} \geqq c$. Indeed if we put $u\left(t_{0}+n\right)=c(1+(1 / n))$, then $u\left(t_{0}+n\right) \downarrow u_{0}=c$. Therefore, when $\alpha=1$, we get that

$$
\begin{array}{cl}
\text { either } & u\left(t_{0}+n_{0}\right) \leqq 0\left(\exists n_{0}\right), \\
& \text { or } u\left(t_{0}+n\right) \downarrow u_{0} \geqq c, \\
\text { or } & u\left(t_{0}+n\right) \uparrow u_{0}>c .
\end{array}
$$

\subsection{Summary of the Section}

Behaviors of $u(t)$ vary greatly according to the initial values $u\left(t_{0}\right)$ and $u\left(t_{0}+1\right)$, which might be seen to be a default of this model. But the model is very interesting because it includes immigration rate $\beta$, in addition to the net growth rate $\alpha$. May be we better consider $\beta$ to depend time $t$ and population $u(t)$, and $\alpha$ would be influenced by $\beta$.

Next we consider complex analytic treatment of the Eq. (1.1), since it admits interesting general solution which would give some prospects in dealing population models.

\section{ANALYTIC SOLUTION}

\subsection{When $\alpha>1$}

We have showed that, if $u\left(t_{0}+n\right)$ is stable, then $u\left(t_{0}+n\right) \rightarrow c$ as $n \rightarrow \infty$. Then $v(t)=u(t)-c$ satisfies $v\left(t_{0}+n\right) \rightarrow 0$ and

$$
\begin{aligned}
v(t+2)= & \alpha v(t+1)+\beta \frac{v(t+1)-v(t)}{\alpha v(t)+\beta} \\
= & \alpha v(t+1)+\frac{v(t+1)-v(t)}{1+(v(t) / c)} \\
= & (\alpha+1) v(t+1)-v(t) \\
& +F(v(t), v(t+1)),
\end{aligned}
$$

where $F\left(v_{1}, v_{2}\right)$ is the sum of terms of higher degree with respect to $v_{1}, v_{2}$. Thus, if $t$ is large and $v(t)$ is small, then $v(t)$ approximately satisfies

$$
v(t+2)-(\alpha+1) v(t+1)+v(t)=0 .
$$

Therefore

$$
u\left(t_{0}+n\right)=c+C_{1} \lambda_{1}^{n}+C_{2} \lambda_{2}^{n}+w\left(t_{0}+n\right),
$$

in which $\lambda_{1}, \lambda_{2}$, are roots of the characteristic equation

$$
\begin{aligned}
& \lambda^{2}-(\alpha+1) \lambda+1=0, \\
& \lambda_{1}=\frac{1}{2}\left(\alpha+1+\sqrt{(\alpha+1)^{2}-4}\right), \quad \lambda_{2}=\frac{1}{\lambda_{1}} .
\end{aligned}
$$


Since $\lambda_{1}>1$, we must have $C_{1}=0$ if we suppose $u(t+n)$ is stable when $n \rightarrow \infty$. Therefore we consider a particular solution of (1.1) in the form

$$
u\left(t_{0}+t\right)=c+\sum_{k=1}^{\infty} a_{k} \lambda_{2}^{k t}
$$

where

$$
0<\lambda=\lambda_{2}=\frac{1}{2}\left(\alpha+1-\sqrt{(\alpha+1)^{2}-4}\right)<1 .
$$

If we suppose $u(t+n)$ is stable when $n \rightarrow-\infty$, then we also consider solutions in the form

$$
u\left(t_{0}+t\right)=c+\sum_{k=1}^{\infty} a_{-k} \lambda_{2}^{-k t} .
$$

Furthermore, if $u\left(t_{0}-n\right) \rightarrow 0(n \rightarrow \infty)$, then we have

$$
\lim _{n \rightarrow-\infty} \frac{u(t+n+2)}{u(t+n+1)}=\alpha
$$

So that we put a formal solution to (1.1) in the form

$$
u(t)=\sum_{k=1}^{\infty} a_{k} \alpha^{k t}
$$

Then $a_{1}$ is arbitrary and $a_{k}=0(k \geqq 2)$. Suppose $x(t)=\phi(t) \alpha^{t}$ be a solution of (1.1) such that $x(t-n) \rightarrow 0$ as $n \rightarrow+\infty$, and $\phi$ satisfies $\lim _{n \rightarrow \infty}\left|\phi(t-n)-a_{1}\right|<M$ uniformly on any compact set for some constant $M$. Then we have

$$
\lim _{n \rightarrow \infty}(\phi(t-n+1)-\phi(t-n))=0 .
$$

\subsection{When $\alpha<1$}

Suppose $u\left(t_{0}+n\right)$ is stable. Suppose $u\left(t_{0}+n\right) \rightarrow c$. Then $v(t)=u(t)-c$ satisfy (3.2), hence $u\left(t_{0}+n\right)$ is written in the form $\left(3.2^{\prime}\right)$, where $\left|\lambda_{j}\right|=1, j=1,2$, which shows that $u\left(t_{0}+n\right)$ cannot tend to $c$. Hence $u\left(t_{0}+n\right)$ is stable but not asymptotically stable.
Furthermore, if $u\left(t_{0}+n\right) \rightarrow 0 \quad(n \rightarrow+\infty)$, then we have

$$
\lim _{n \rightarrow+\infty} \frac{u(t+n+2)}{u(t+n+1)}=\alpha
$$

So that we put a formal solution to (1.1) in the form

$$
u(t)=\sum_{k=1}^{\infty} a_{k} \alpha^{k t} .
$$

Then $a_{1}$ is arbitrary and $a_{k}=0(k \geqq 2)$. Suppose $x(t)=\phi(t) \alpha^{t}$ be a solution of (1.1) such that $x(t+n) \rightarrow 0 \quad$ as $\quad n \rightarrow+\infty$, and $\phi \quad$ satisfies $\lim _{n \rightarrow \infty}\left|\phi(t+n)-a_{1}\right|<M$ uniformly on any compact set for some constant $M$. Then we have

$$
\lim _{n \rightarrow \infty}(\phi(t+n+1)-\phi(t+n))=0 .
$$

\subsection{When $\alpha=1$}

Suppose $u\left(t_{0}+n\right) \uparrow u_{0}$ or $u\left(t_{0}+n\right) \downarrow u_{0}$ with $u_{0}>c$, then

$$
\frac{u\left(t_{0}+n+2\right)-u\left(t_{0}+n+1\right)}{u\left(t_{0}+n+1\right)-u\left(t_{0}+n\right)} \rightarrow \frac{c}{u_{0}}<1 .
$$

Hence we get

$$
u\left(t_{0}+t+1\right)-u\left(t_{0}+t\right)=\sum_{k=1}^{\infty} d_{k}\left(\frac{c}{u_{0}}\right)^{k t}
$$

and $u\left(t_{0}+t\right)$ will be obtained.

\section{EXISTENCE OF ANALYTIC STABLE SOLUTIONS IN THE CASE $\alpha>1$}

In this section, we write $u\left(t_{0}+t\right)$ simply as $u(t)$. Time $t$ is of course a real variable. But in this section, we consider $t$ to be a complex variable, and we will prove existence of analytic solutions.

First we shall show existence of a particular solution of (3.1). 
When $\alpha>1$, we can determine a formal solution to (3.1) in the form (4.1)

$$
v(t)=\sum_{n=1}^{\infty} a^{n} \lambda^{n t},
$$

where $0<\lambda=\lambda_{2}<1$.

$$
\begin{aligned}
a_{n} \beta & \left\{\lambda^{2 n}-(1+\alpha) \lambda^{n}+1\right\} \\
=\alpha & \alpha \sum_{k=1}^{n-1} a_{k} a_{n-k} \lambda^{n-k}\left(\alpha-\lambda^{n-k}\right),\left(a_{1}: \text { arbitrary }\right)
\end{aligned}
$$

\subsection{Make a Map $T$ and Existence of a Fixed Point of $T$}

We rewrite (3.1) as

$$
v(t)=\beta \frac{(1+\alpha) v(t+1)-v(t+2)}{\alpha v(t+2)-\alpha^{2} v(t+1)+\beta}
$$

which is written as

$$
v(t)=f(v(t+1), v(t+2))
$$

where

$$
f\left(v_{1}, v_{2}\right)=\beta \frac{(1+\alpha) v_{1}-v_{2}}{\alpha v_{2}-\alpha^{2} v_{1}+\beta} .
$$

Let $N$ be a positive integer. Put $P_{N}(t)=$ $\sum_{n=1}^{N} a_{n} \lambda^{n t}$ and $y(t)=v(t)-P_{N}(t)$. Then by (4.2) we obtain

$$
\begin{aligned}
y(t)= & \\
f( & \left.y(t+1)+P_{N}(t+1), y(t+2)+P_{N}(t+2)\right) \\
& -P_{N}(t) \\
= & g_{1}(t, y(t+1), y(t+2))+g_{2}(t) \\
= & g(t, y(t+1), y(t+2)),
\end{aligned}
$$

in which

$$
\begin{aligned}
& g_{1}(t, y(t+1), y(t+2)) \\
& \quad=f\left(y(t+1)+P_{N}(t+1), y(t+2)+P_{N}(t+2)\right) \\
& \quad-f\left(P_{N}(t+1), P_{N}(t+2)\right), \\
& g_{2}(t)=f\left(P_{N}(t+1), P_{N}(t+2)\right)-P_{N}(t) .
\end{aligned}
$$

Put

$$
\begin{aligned}
S(\rho)= & \left\{t \in \mathbb{C}:\left|\lambda^{t}\right| \leq \rho\right\} \\
H(A, \rho)= & \{y: y(t) \text { is holomorphic and } \\
& \left.|y(t)| \leqq A\left|\lambda^{t}\right|^{N+1} \text { for } t \in S(\rho)\right\} .
\end{aligned}
$$

Take $A>0$ and $0<\rho<1$, which will be determined later. For $y(t) \in H(A, \rho)$, put

$$
\begin{aligned}
T[y](t) & =g(t, y(t+1), y(t+2)) \\
& =g_{1}(t, y(t+1), y(t+2))+g_{2}(t) .
\end{aligned}
$$

We will show that, constants $A$ and $\rho$ may be chosen so that $T$ maps $H(A, \rho)$ into $H(A, \rho)$.

We note that, if $\left|v_{1}\right|,\left|v_{2}\right|<\beta /\left(4 \alpha^{2}\right)$, then

$$
\left|\frac{\partial f}{\partial v_{1}}\right|,\left|\frac{\partial f}{\partial v_{2}}\right| \leqq 8(1+\alpha) \text {. }
$$

Since

$$
\begin{aligned}
g_{1}( & t, y(t+1), y(t+2)) \\
= & \int_{0}^{1} \frac{\mathrm{d}}{\mathrm{d} s} f\left(s y(t+1)+P_{N}(t+1), s y(t+2)\right. \\
& \left.+P_{N}(t+2)\right) \mathrm{d} s \\
= & \int_{0}^{1}\left\{y(t+1) \frac{\partial f}{\partial v_{1}}(*)+y(t+2) \frac{\partial f}{\partial v_{2}}(*)\right\},
\end{aligned}
$$

where $(*)=s y(t+1)+P_{N}(t+1), s y(t+2)+P_{N}(t+2)$, if we suppose

$$
\begin{aligned}
& \left|s y(t+1)+P_{N}(t+1)\right|,\left|s y(t+2)+P_{N}(t+2)\right| \\
& \quad<\frac{\beta}{4 \alpha^{2}},
\end{aligned}
$$

then we have

$$
\begin{aligned}
& \left|g_{1}(t, y(t+1), y(t+2))\right| \\
& \quad \leqq \int_{0}^{1}(|y(t+1)|+|y(t+2)|) \cdot 8(1+\alpha) \mathrm{d} s \\
& \quad \leqq 16(1+\alpha)\left\{A\left|\lambda^{t}\right|^{N+1} \cdot|\lambda|^{N+1}\right\} .
\end{aligned}
$$

Suppose $N$ is so large such that

$$
16(1+\alpha)|\lambda|^{N+1} \leqq \frac{1}{4},
$$


then we have, by (4.8)

$$
\left|g_{1}(t, y(t+1), y(t+2))\right| \leqq \frac{1}{4} A\left|\lambda^{t}\right|^{N+1},
$$

if (4.7) holds.

We take sufficiently small $\delta$ such that

$|y(t+1)|,|y(t+2)|,\left|P_{N}(t+1)\right|,\left|P_{N}(t+2)\right|<\frac{\beta}{8 \alpha^{2}}$

Then we have (4.7), hence obtain (4.10).

Since (4.1) is a formal solution of (4.2), we have

$$
\left|g_{2}(t)\right| \leqq K_{2}\left|\lambda^{t}\right|^{N+1}
$$

with a constant $K_{2}$ which depends on $N$. Suppose $N$ is so large that (4.9) holds, and $A$ is so large that

$$
\frac{1}{4} A+K_{2}<\frac{1}{2} A
$$

i.e.,

$$
A>2 K_{2}
$$

and $\rho$ is so small that (4.7) holds, then we obtain that $T$ in (4.4) maps $H(A, \rho)$ into itself.

The map $T$ is obviously continuous if $H(A, \rho)$ is endowed with the topology of uniform on convergence $S(\rho)$.

$H(A, \rho)$ is clearly convex, and is compact by the theorem of Montel [5].

Thus by Schauder's fixed point theorem in [5], we obtain the existence of a fixed point $y(t)=y_{N}(t) \in$ $H(A, \rho)$ of $T$.

\subsection{Uniqueness of the Fixed Point}

Suppose there is another fixed point $y^{*}(t)=y_{N}^{*}(t) \in$ $H\left(A^{*}, \rho^{*}\right)$. Put

$$
\begin{gathered}
A_{0}=\max \left(A, A^{*}\right), \quad \rho \leqq \min \left(\rho, \rho^{*}\right), \\
v(t)=y_{N}(t)+P_{N}(t), \\
v^{*}(t)=y_{N}^{*}(t)+P_{N}(t),
\end{gathered}
$$

and

$$
w(t)=y_{N}(t)-y_{N}^{*}(t)
$$

From (4.3), we have

$w(t)$

$$
\begin{aligned}
=\{ & f\left(y_{N}^{*}(t+1)+P_{N}(t+1), y_{N}^{*}(t+2)+P_{N}(t+2)\right) \\
& \left.-P_{N}(t)\right\} \\
-\{ & f\left(y_{N}(t+1)+P_{N}(t+1), y_{N}(t+2)+P_{N}(t+2)\right) \\
& \left.-P_{N}(t)\right\} \\
= & f\left(w(t+1)+v_{N}(t+1), w(t+2)+v_{N}(t+2)\right) \\
& -f\left(v_{N}(t+1), v_{N}(t+2)\right) \\
= & \int_{0}^{1}\left\{w(t+1) \frac{\partial f}{\partial v_{1}}(* *)+w(t+2) \frac{\partial f}{\partial v_{2}}(* *)\right\} \mathrm{d} s,
\end{aligned}
$$

where $\quad(* *)=\left(s w(t+1)+v_{N}(t+1), \quad s w(t+2)+\right.$ $\left.v_{N}(t+2)\right)$.

If $\rho_{0}$ is sufficiently small, then

$$
\left|\frac{\partial f}{\partial v_{1}}(* *)\right|,\left|\frac{\partial f}{\partial v_{2}}(* *)\right|<8(1+\alpha),
$$

and

$$
32(1+\alpha)|\lambda|^{N+1}<\frac{1}{2}
$$

Thus we have

$$
\begin{aligned}
|w(t)| & <8(1+\alpha)(|w(t+1)|+|w(t+2)|) \\
& \leqq 8(1+\alpha)\left(2 A_{0}\left|\lambda^{t+1}\right|^{N+1}+2 A_{0}\left|\lambda^{t+2}\right|^{N+1}\right) \\
& <32(1+\alpha)|\lambda|^{N+1} \cdot A_{0}\left|\lambda^{t}\right|^{N+1} \\
& <\frac{1}{2} A_{0}\left|\lambda^{t}\right|^{N+1} .
\end{aligned}
$$

Then

$$
\left|v(t)-v^{*}(t)\right| \leqq \frac{1}{2} A_{0}\left|\lambda^{t}\right|^{N+1}=\frac{1}{4}\left(2 A_{0}\right)\left|\lambda^{t}\right|^{N+1},
$$

for $t \in S\left(\rho_{0}\right)$. 
Repeating this procedure $k$ times we obtain

$$
\begin{aligned}
& \left|v(t)-v^{*}(t)\right|<\left(\frac{1}{4}\right)^{k}\left(2 A_{0}\right)\left|\lambda^{t}\right|^{N+1}, \\
& \quad k=1,2, \ldots
\end{aligned}
$$

Letting $k \rightarrow \infty$, we have

$$
v(t)=v^{*}(t), \quad t \in S\left(\rho_{0}\right) .
$$

Thus $v(t)$ and $v^{*}(t)$ are holomorphic in $\left|\lambda^{t}\right| \leqq$ $\min \left(\rho, \rho^{*}\right)$ and $v(t) \equiv v^{*}(t)$ there.

\subsection{Arbitrariness of $N$ (Independence of $N$ )}

Finally we will show that the solution $y_{N}(t)$ does not depend on $N$, which shows that (4.1) is actually a solution.

Let $y_{N}(t) \in H\left(A_{N}, \rho_{N}\right)$ and $y_{N+1}(t) \in H\left(A_{N+1}\right.$, $\left.\rho_{N+1}\right)$ be fixed points, and

$$
\begin{aligned}
v_{N+1}(t) & =y_{N+1}(t)+P_{N+1}(t) \\
& =y_{N+1}(t)+a_{N+1} \lambda^{(N+1) t}+P_{N}(t) \\
& =\tilde{y}_{N}(t)+P_{N}(t) .
\end{aligned}
$$

Obviously

$$
\begin{aligned}
\tilde{y}_{N}(t) & =\left|y_{N+1}(t)+a_{N+1} \lambda^{(N+1) t}\right| \\
& \leqq A_{N+1} \lambda^{N+2}\left|\lambda^{t}\right|^{N+2}+\left|a_{N+1}\right| \cdot\left|\lambda^{t}\right|^{N+1} \\
& =A_{N}^{*}\left|\lambda^{t}\right|^{N+1} .
\end{aligned}
$$

By uniqueness of fixed point, $\tilde{y}_{N}(t)=y_{N}(t)$ for $t \in S\left(\rho_{N}\right) \cap S\left(\rho_{N+1}\right)$. Thus

$$
v_{N}(t)=v_{N+1}(t) \quad \text { in } S\left(\rho_{N}\right) \cap S\left(\rho_{N+1}\right) .
$$

By analytic prolongation, both of $v_{N}(t)$ and $v_{N+1}(t)$ are holomorphic in $S\left(\rho_{N}\right) \cap S\left(\rho_{N+1}\right)$ and coincide there.

Thus we have proved that a solution $v(t)$ is defined and holomorphic in $S(\rho)$ for a $\rho>0$, which has the expansion (4.1). By analytic prolongation, the solution $v(t)$ is defined on the whole plane by using the Eq. (4.12)

$$
v(t-2)=\frac{\alpha \beta\{(1+\alpha) v(t-1)-v(t)\}}{\alpha v(t)-\alpha^{2} v(t-1)+\beta} .
$$

\subsection{Analytic General Solutions}

Analytic solutions of some difference equations are investigated in [6-9]. In this section, we shall investigate analytic general solutions of (1.1).

From [7], we have the following theorem:

THEOREM 1 Let $v(s)$ be the solution of (4.2) obtained in Section 3. Suppose $x(t)$ be an analytic solution of (4.2) such that $x(t-n) \rightarrow 0$ as $n \rightarrow+\infty$, uniformly on any compact set. Then there is a periodic entire function $\pi(t),(\pi(t+1)=\pi(t))$, such that

$$
x(t)=v\left(t+\frac{\log \pi(t)}{\log \lambda}\right) .
$$

Conversely if we put

$$
x(t)=v\left(t+\frac{\log \pi(t)}{\log \lambda}\right),
$$

where $\pi$ is a periodic function whose period is one, then $x(t)$ is a solution of (3.1).

Now we have a general solution of (3.1) such that

$$
x(t)=v\left(t+\frac{\log \pi(t)}{\log \lambda}\right),
$$

where $\pi$ is an arbitrarily periodic function whose period is one. And we have sought general solutions of the population model which is given by the Eq. (1.1) such that

$$
u(t)=\frac{\beta}{\alpha}+v\left(t+\frac{\log \pi(t)}{\log \lambda}\right)
$$

where $\pi(t)$ is an arbitrarily periodic function whose period is one. 


\section{References}

[1] Jacques Bélair, Population models with state-dependent delays. Mathematical population dynamics, Lecture Notes in Pure and Applied Mathematics, 131 (1991), 165-176, Marcel Dekker, New York-basel-Hong Kong.

[2] D. Dendrinos, Private communication.

[3] W.J. Evens, Mathematical Population Genetics. Biomathematics, Springer-Verlag, Berlin-Heidelberg-New York, 1979.

[4] J.D. Murray, Mathematical Biology. Biomathematics Texts, Springer-Verlag, Berlin-Heidelberg-New York, 1989.
[5] D.R. Smart, Fixed Point Theorems, Cambridge University, Press, 1974.

[6] M. Suzuki, Holomorphic solutions of some functional equations, Nihonkai Mathematical Journal, 5 (1994), $109-114$.

[7] M. Suzuki, On some difference equations in economic model, Mathematica Japonica, 43 (1996), 129-134.

[8] M. Suzuki, Holomorphic solutions of some system of $n$ functional equations with $n$ variables related to difference systems, Aequationes Mathematicae, 57 (1999), 21-36.

[9] N. Yanagihara, Meromorphic solutions of some difference equations, Funkcial Ekvac, 23 (1980), 309-326. 


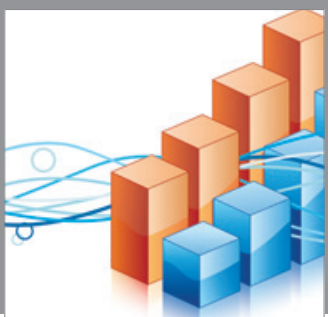

Advances in

Operations Research

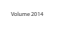

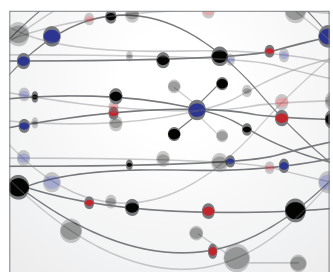

\section{The Scientific} World Journal
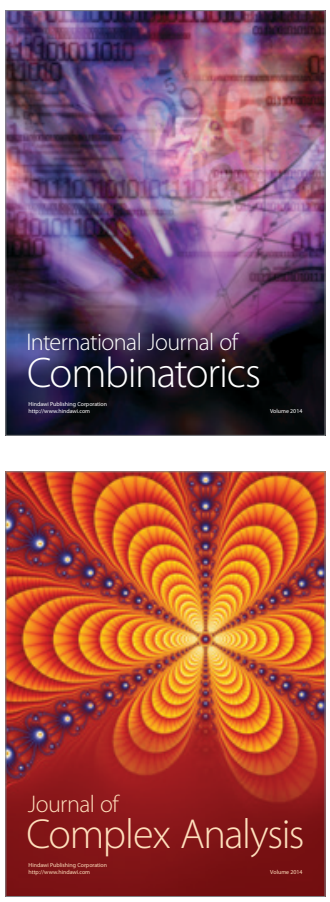

International Journal of

Mathematics and

Mathematical

Sciences
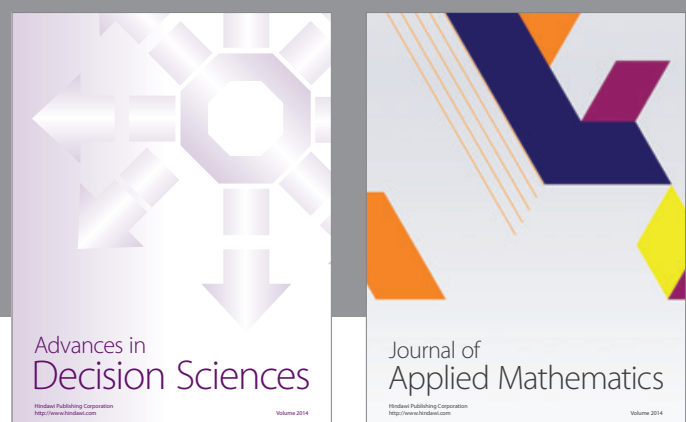

Journal of

Applied Mathematics
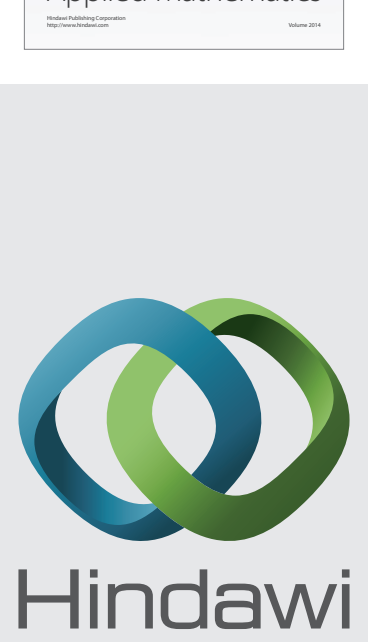

Submit your manuscripts at http://www.hindawi.com
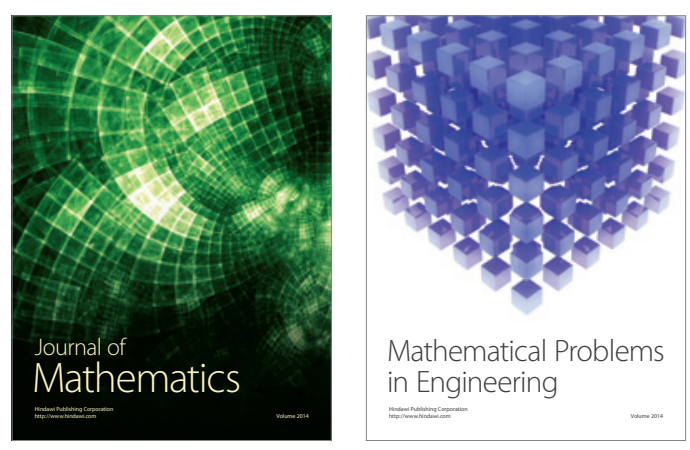

Mathematical Problems in Engineering
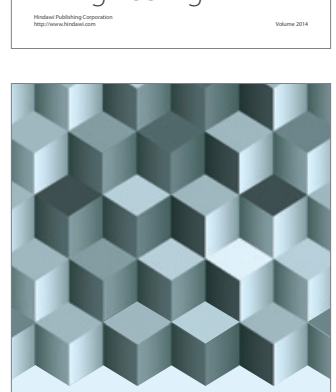

Journal of

Function Spaces
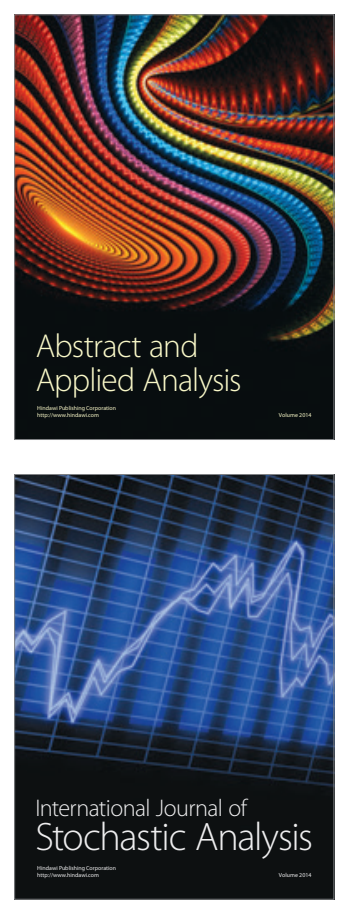

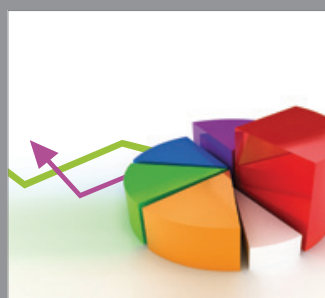

ournal of

Probability and Statistics

Promensencen
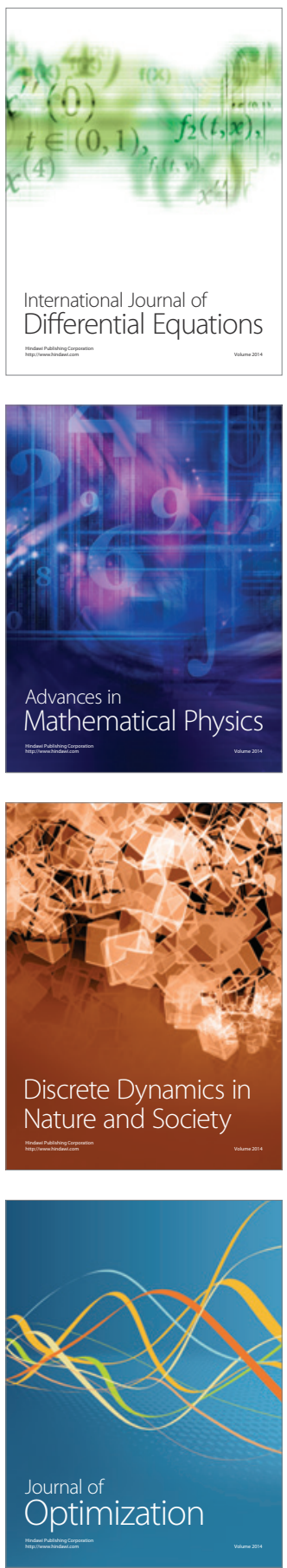